\title{
LOCAL COMPLETENESS OF OPERATOR ALGEBRAS
}

\author{
H. BEHNCKE AND J. CUNTZ
}

\begin{abstract}
A normed *-algebra $\mathcal{Q}$ is called a local $C^{*}$-algebra, if all its maximal commutative *-subalgebras are $C^{*}$-algebras. It is shown that any local $C^{*}$-algebra dense in $\mathcal{K}(\mathcal{X})$, the algebra of compact operators on the Hilbert space $\mathcal{K}$, equals $\mathcal{K}(\mathcal{K})$. The same result holds also for local $C^{*}$ algebras dense in $A W^{*}$-algebras without a $\mathrm{II}_{1}$ summand.
\end{abstract}

A local property of an involutive algebra $\mathcal{Q}$ is a property determined by or on its commutative star subalgebras. In recent years a number of papers have been devoted to the study of global properties of Banach star algebras which are implied by corresponding local properties. As examples we mention the connection between hermiticity and symmetry of involutive Banach algebras by Shirali and Ford, diverse characterizations of $C^{*}$-algebras [3], or the characterization of $W^{*}$-algebras by Pedersen in [8]. An example of a global property which is implied by the corresponding local property is also the continuity of a linear functional on $C^{*}$-algebras [6], [9].

The present paper is another attempt to weaken the axioms of $C^{*}$-algebras. At the same time we obtain some insight into the structure of (certain) $C^{*}$. algebras.

Definition 1. A complex normed algebra $\mathbb{Q}$ with involution is a local $C^{*}$ algebra, if all of its closed commutative star subalgebras are $C^{*}$-algebras with the given norm and involution.

Let $|\cdot|$ be the given norm on $\mathcal{Q}$. Then $\|\cdot\|$ defined by $\|x\|=\max \left\{|x|,\left|x^{*}\right|\right\}$ is an algebra-norm on $\mathbb{Q}$ which makes the involution isometric. The involution can, in a natural way, be extended to an involution on the $\|\cdot\|$-completion $\tilde{\mathcal{Q}}$ of Q.

Now let $x=x^{*} \in \tilde{\mathbb{Q}}$ and let $\mathscr{B}$ be the closed star subalgebra of $\tilde{\mathbb{Q}}$ generated by $x$. There is a sequence $x_{n}=x_{n}^{*} \in \mathbb{Q}$ with $x_{n} \rightarrow x$. If $p$ is any complex polynomial without constant coefficient, then $p\left(x_{n}\right) \rightarrow p(x)$ and by continuity we get $\left\|p(x) p(x)^{*}\right\|=\|p(x)\|^{2}$.

Because of the density of such elements we have more generally $\left\|y y^{*}\right\|$ $=\|y\|^{2}$ for all $y \in \mathscr{B}$. Thus $\mathscr{B}$ is a $C^{*}$-algebra and, in particular, $x$ has real spectrum and $\tilde{\mathbb{Q}}$ has hermitean involution. Arguing now as in [1] or [3, Chapter

Received by the editors December 29, 1975.

AMS (MOS) subject classifications (1970). Primary 46L05. 
41], let $\|a\|=\rho\left(a^{*} a\right)^{1 / 2}=\left\|a^{*} a\right\|^{1 / 2}$ be the greatest $C^{*}$-seminorm on $\tilde{\mathcal{Q}}$. For all $a \in \mathfrak{Q}$ we have $\|a\|^{2}=\left\|a^{*} a\right\| \leqslant\|a\|^{2}$ and

$$
\left.\|a\| \leqslant\left[\left\|a+a^{*}\right\|+\left\|a-a^{*}\right\|\right] / 2=\left[\left\|a+a^{*}\right\| \mid+\left\|a-a^{*}\right\|\right]\right] / 2 \leqslant 2\|a\| \|,
$$

so that $\|\cdot\| \cdot \|$ and $\|\cdot\|$ are equivalent on $\tilde{\mathscr{e}}$, and $\tilde{\mathcal{Q}}$ equipped with $\|\cdot\| \|$ is a $C^{*}$. algebra.

We may extend $|\cdot|$ by continuity to an algebra seminorm of $\tilde{\mathcal{e}}$. Then $|\cdot|$ is in fact a norm, since if $|a|=0$ we get $\left|a^{*} a\right|=\left\|a^{*} a\right\|=\left\|a^{*} a\right\|=0$ whence $a=0$.

By a theorem of Cleveland $[4,5.4]$ any algebra-norm on a $C^{*}$-algebra majorizes a multiple of the $C^{*}$-norm. Hence $|\cdot|$ and $\|\cdot|\||$ are equivalent on $\tilde{\mathcal{Q}}$. Naturally, one would like to show that every local $C^{*}$-algebra is complete. Thus, for this purpose there is no loss of generality in considering only local $C^{*}$-algebras $\mathbb{Q}$ which are dense subalgebras of a $C^{*}$-algebra $\tilde{\mathcal{E}}$ and are equipped with the norm inherited from $\tilde{\mathcal{E}}$. An easy argument shows that it suffices to solve this problem for separable algebras with identity only. Certain questions related to local $C^{*}$-algebras have been considered in [5] and [6]. Specifically it has been shown that a local $C^{*}$-algebra which is a Banach algebra in some norm is in fact complete. In the present paper, however, we do not assume that $\mathbb{Q}$ is complete in any norm. The question whether a local $C^{*}$-algebra $\mathbb{Q}$ is complete is closely related to the structure of $\tilde{\mathbb{Q}}$.

Definition 2. A $C^{*}$-algebra $\tilde{\mathcal{e}}$ is called interwoven if the only local $C^{*}$ algebra dense in $\tilde{e}$ is $\tilde{e}$ itself.

We show that certain classes of $C^{*}$-algebras are interwoven. Our first result is: The algebra $\mathscr{K}(\mathcal{H})$ of all compact operators on a Hilbert space $\mathcal{H}$ is interwoven. This result extends to dual $C^{*}$-algebras and to separable $C^{*}$. algebras with a finite dual. Theorem 2 states that $C^{*}$-algebras which are in a certain sense infinite, are interwoven. The main consequence of this theorem is that $\mathscr{B}(\mathcal{H})$ and more generally all properly infinite $A W^{*}$-algebras are interwoven.

Lemma 1. Let $\mathbb{Q}$ be a local $C^{*}$-algebra dense in the $C^{*}$-algebra $\tilde{\mathbb{Q}}$ and let $0<\varepsilon<1$. For any projection $P \in \mathbb{Q}$ there exists a projection $Q \in \mathbb{Q}$ such that $|P-Q|<\varepsilon$. If $P$ is central, then $P \in \mathbb{Q}$.

Proof. Let $x=x^{*} \in \mathbb{Q}$ with $|x-P|<\varepsilon / 2$. Then the spectrum of $x$ lies in $(-\varepsilon / 2, \varepsilon / 2) \cup(1-\varepsilon / 2,1+\varepsilon / 2)$. There exists a function $f$ continuous on this set, such that $Q=f(x)$ is a projection with $|f(x)-x|<\varepsilon / 2$ or $|f(x)-P|<\varepsilon$. If $P$ is central, then $P Q=Q P$, whence $P=Q$.

We say that two projections $E$ and $F$ are equivalent in $\mathbb{Q}(E \sim F$ in $\mathbb{Q})$, if there is a partial isometry $U$ in $\mathbb{Q}$ with $U^{*} U=E$ and $U U^{*}=F$.

Lemma 2. If two projections $P, Q \in \mathbb{Q}$ are equivalent in $\tilde{\mathcal{Q}}$, they are equivalent in $Q$. 
Proof. Let $V$ be a partial isometry in $\tilde{\mathcal{Q}}$ with $V^{*} V=P$ and $V V^{*}=Q$. Choose $W \in \mathbb{Q}$ with $|W-V|<1 / 4$ such that $W=Q W P$. Then $\mid W^{*} W$ $-V^{*} V|\leqslant| W^{*} W-W^{*} V|+| W^{*} V-V^{*} V \mid<\frac{5}{16}+\frac{1}{4}<\frac{3}{4}$. Since, moreover, $P\left(W^{*} W\right)=\left(W^{*} W\right) P=W^{*} W$, there is an element $A$ in the $C^{*}$ algebra generated by $W^{*} W$ such that $P=\left(W^{*} W\right)^{1 / 2} A$. Then $U=W A$ is a partial isometry in $\mathbb{Q}$ with the required properties.

Lemma 3. If $\mathcal{G}$ is a closed $*$-ideal in $\tilde{\mathbb{Q}}$, then $\mathbb{Q} \cap \mathcal{G}$ is dense in $\mathscr{G}$. If $\mathscr{G}$ is interwoven, then $\mathscr{G} \subset \mathbb{Q}$.

Proof. Let $x=x^{*} \in \mathcal{G}, x \geqslant 0$, and let $y_{n}=y_{n}^{*} \in \mathbb{Q}, y_{n} \geqslant 0$, such that $\left|x-y_{n}\right|<1 / n$. Let $f_{n}: \mathbf{R} \rightarrow \mathbf{R}$ be the continuous function defined by $f_{n}(t)=0$ if $t \leqslant 1 / n ; f_{n}(t)=t$; if $t \geqslant 2 / n ; f_{n}$ is linear on $[1 / n, 2 / n]$ and let $\Psi: \tilde{\mathbb{Q}} \rightarrow \tilde{\mathbb{Q}} / \mathcal{G}$ be the canonical homomorphism. Then, since $\left|\Psi\left(y_{n}\right)\right| \leqslant 1 / n$, we get $\Psi\left(f_{n}\left(y_{n}\right)\right)$ $=f_{n}\left(\Psi\left(y_{n}\right)\right)=0$ or $f_{n}\left(y_{n}\right) \in 9$. Since $\left|y_{n}-f_{n}\left(y_{n}\right)\right|<1 / n$, we get $\left|x-f_{n}\left(y_{n}\right)\right|$ $<2 / n$. The remainder follows from the fact that $Q \cap g$ is a local $C^{*}$-algebra.

THEOREM 1. $\mathcal{K}(\mathcal{H C})$ is interwoven.

Proof. Let $Q$ be a local $C^{*}$-algebra which is dense in $\mathscr{K}(\mathcal{K})$. Because of the spectral theorem it suffices to show that $\mathscr{Q}$ contains every rank one projection. For simplicity we say that a vector in $\mathcal{H}$ is attainable if the corresponding projection belongs to $\mathcal{Q}$. It follows from Lemma 2 that finite linear combinations of attainable vectors are attainable $(\mathscr{Q}$ contains the full algebra of linear operators on the subspace generated by these vectors).

(a) Let $P_{0}, P_{1}, P_{2}, \ldots$ be orthogonal rank one projections in $\mathcal{Q}$ with normalized range vectors $e_{0}, e_{1}, e_{2}, \ldots$ We show that every vector in $P(\mathcal{H})$, where $P=\sum_{n=0}^{\infty} P_{n}$, is attainable. For the proof, let $\lambda_{i} \geqslant 0$ and $\sum_{i=1}^{\infty} \lambda_{i}^{2}<1 / 4$.

Consider the vectors

$$
f_{n}=\lambda_{n} e_{0}+\sum_{k=1}^{n-1} \alpha_{k}^{(n)} e_{k}+e_{n}, \quad n=1,2, \ldots
$$

These vectors are attainable. They are orthogonal, if one chooses

$$
\alpha_{k}^{(n)}=-\lambda_{n} \lambda_{k} \beta_{k} \text { for } n>k,
$$

where $\beta_{1}=1$ and

$$
\beta_{k+1}=1+\sum_{l=1}^{k} \lambda_{l}^{2} \beta_{l}^{2}
$$

For this choice of coefficients one gets $0 \leqslant \beta_{k} \leqslant 2$ and $\left|f_{n}\right| \leqslant 1+2 \lambda_{n}^{2}$.

Let $Q_{n}$ be the projection onto the subspace generated by $f_{n}$. Then $Q_{n} \in \mathbb{Q}$ and also $A=\sum_{n=1}^{\infty} \mu_{n}\left|f_{n}\right|^{2} Q_{n} \in \mathbb{Q}$ for each sequence $\mu_{n} \rightarrow 0$. Since $A P_{0} A^{*}$ $\in \mathbb{Q}$ is a rank one hermitean operator, it is a multiple of the projection onto its range. Hence the range vector of $A P_{0}$, the vector $g=\sum_{n=1}^{\infty} \mu_{n} \lambda_{n} f_{n}$ is attainable.

However we have 


$$
g=\left(\sum_{n=1}^{\infty} \lambda_{n}^{2} \mu_{n}\right) e_{0}+\sum_{l=1}^{\infty}\left[\mu_{l}-\sum_{n>l} \mu_{n} \lambda_{n}^{2} \beta_{l}\right] \lambda_{l} e_{l}
$$

choosing $0<\mu_{n} \searrow 0$ we get

$$
g=\left(\sum_{n=1}^{\infty} \lambda_{n}^{2} \mu_{n}\right) e_{0}+\sum_{l=1}^{\infty} \lambda_{l} \mu_{l}\left(1-\gamma_{l}\right) e_{l} \quad \text { with } \gamma_{l} \rightarrow 0 .
$$

Hence the vector

$$
h=\sum_{l=1}^{\infty} \lambda_{l} \mu_{l}\left(1-\gamma_{l}\right) e_{l}
$$

is attainable. Moreover, the vector

$$
\left(\sum_{n=1}^{\infty} \gamma_{n} /\left(1-\gamma_{n}\right) P_{n}\right)(h)=\sum_{n=1}^{\infty} \lambda_{n} \mu_{n} \gamma_{n} e_{n}
$$

is attainable. Then also $\sum_{n=1}^{\infty} \lambda_{n} \mu_{n} e_{n}$ is attainable.

Since finite linear combinations of attainable vectors are attainable and since any $l^{2}$-sequence can be written as the product of an $l^{2}$-sequence and a monotone $c_{0}$-sequence, this shows that every vector in $P(\mathcal{H})$ is attainable.

(b) Now let $\mathscr{B}$ be a maximal abelian star subalgebra of $\mathscr{Q}$ with minimal projections $\left\{P_{\alpha}\right\}$. Let $Q=\sum P_{\alpha}$. It follows easily from (a), that every vector in $Q(\mathcal{H})$ is attainable. It remains to show, that $Q=1$. If $Q<1$, there is a onedimensional projection $E$ in $\mathcal{K}(\mathcal{K})$ with $E Q=0$. Choose a projection $F \in \mathbb{Q}$ such that $|E-F|<\varepsilon \leqslant 1 / 4$ (Lemma 1). An easy exercise in analytic geometry shows that $Q, E$ and $F$ have a representation:

$$
\begin{aligned}
& \mathcal{K}=\mathcal{H}_{0} \oplus \mathbf{C} \oplus \mathbf{C}, \quad Q=Q_{0} \oplus\left(\begin{array}{ll}
1 & 0 \\
0 & 0
\end{array}\right), \\
& E=0 \oplus\left(\begin{array}{ll}
0 & 0 \\
0 & 1
\end{array}\right) \text { and } F=0 \oplus\left(\begin{array}{ll}
c^{2} & c s \\
c s & s^{2}
\end{array}\right)
\end{aligned}
$$

with $c, s \in \mathbf{R}, c^{2}+s^{2}=1$ and $c^{2}<\varepsilon$. By (a) we have $Q F Q \in \mathbb{Q}$. Hence $s^{2} F-(F-Q F Q)^{2} \in \mathbb{Q}$. This expression, however, is orthogonal to $Q$ and nonzero, in contradiction to the maximality of $\mathscr{B}$.

Corollary 1. Every dual $C^{*}$-algebra $C$ is interwoven.

Proof. $C$ is the weak $C^{*}$-sum of algebras $\mathscr{K}_{i}$, where each $\mathcal{K}_{i}$ is isomorphic to the algebra of compact operators on some Hilbert space $\mathcal{T}_{i}$. If $\mathbb{Q}$ is a local $C^{*}$-algebra which is dense in $\mathcal{C}$, then by Lemma 3 we have $\mathcal{K}_{i} \subset \mathbb{Q}$ for all $i$. From this $\mathbb{Q}=\mathcal{C}$ follows easily.

COROLlary 2. Every separable $C^{*}$-algebra $\mathcal{C}$ with a finite dual $\hat{e}$ is interwoven.

Proof. Use induction on the number of points in $\hat{C}$ and the fact that $\mathcal{C}$ is a splitting extension of its maximal liminal ideal which is dual [2]. 
THEOREM 2. Let $C$ be a $C^{*}$-algebra with unit 1 that contains a projection $E$ such that $E \sim(1-E) \sim 1$ in $\mathcal{C}$. Then $\mathcal{C}$ is interwoven.

Proof. Let $\mathscr{Q}$ be a local $C^{*}$-algebra which is dense in $\mathcal{C}$. By Lemma 1 there is a projection $F$ in $\mathscr{Q}$ such that $|E-F|<1$. Using Lemma 2 we see $F \sim(1-F) \sim 1$ in $\mathbb{Q}$. Let $V_{1}, V_{2} \in \mathbb{Q}$ such that

$$
V_{1}^{*} V_{1}=1, \quad V_{1} V_{1}^{*}=F \quad \text { and } \quad V_{2}^{*} V_{2}=F, \quad V_{2} V_{2}^{*}=1-F
$$

Define partial isometries $U_{1}, U_{2}, U_{3}$ and a projection $P$ in $Q$ by

$$
U_{1}=V_{1} F, \quad U_{2}=V_{2} U_{1} V_{2}^{*}, \quad P=V_{1}(1-F) V_{1}^{*}, \quad U_{3}=V_{2} P .
$$

Then $U=U_{1}+U_{2}^{*}+U_{3}^{*}$ is a unitary element of $\mathcal{Q}$ and $\left\{U^{n} P U^{-n} \mid n \in \mathbf{N}\right\}$ is a set of mutually orthogonal projections in $Q_{\text {. }}\left(U_{1}\right.$ may be interpreted as a unilateral shift with infinite multiplicity on the "subspace corresponding to $F$ " and $P=F-U_{1} U_{1}^{*}$. Then $U$ is a bilateral extension of $U_{1}$.) Since $P$

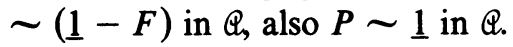

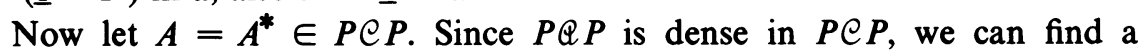
sequence $\left\{A_{n}\right\}_{n \in \mathrm{N}}$ of selfadjoint elements in $P Q P$ with $\left|A_{i}\right| \rightarrow 0$ and an $l^{1}$ sequence $\left\{\lambda_{n}\right\}_{n \in \mathrm{N}}$ of positive real numbers such that $A=\sum_{n=1}^{\infty} \lambda_{n}^{2} A_{n}$.

The elements $U^{n} A_{n} U^{-n}(n \in \mathrm{N})$ are pairwise orthogonal and selfadjoint, therefore their sum $x=\sum_{n \in \mathbf{N}} U^{n} A_{n} U^{-n}$ lies in $Q$. Since $U$ admits functional calculus in $Q$, also $y=\sum_{n=1}^{\infty} \lambda_{n} U^{n}$ is in $\mathcal{Q}$. Hence $A=P y^{*} x y P$ is in $\mathbb{Q}$. Thus

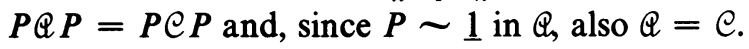

Corollary 3. (a) Every $C^{*}$-algebra of the form $\mathcal{C}=\mathscr{B}(\mathcal{H C}) \otimes \mathcal{C}^{\prime}$, where $\mathcal{C}^{\prime}$ is $a C^{*}$-algebra with unit and $\mathcal{H}$ an infinite-dimensional Hilbert space, is interwoven.

(b) Every $A W^{*}$-algebra $\mathcal{C}$ without a type $\mathrm{II}_{1}$ direct summand is interwoven.

Proof. (a) is an immediate consequence of Theorem 2. To prove (b) let the local $C^{*}$-algebra $\mathcal{Q}$ be dense in $\mathcal{C}$. Because of Lemma 1 the centre of $\mathcal{C}$ belongs to $\mathcal{C}$. Hence we may consider the finite and the infinite part of $\mathcal{C}$ separately. The properly infinite part $\mathfrak{N}$ of $\mathcal{C}$ contains a projection $E$ such that $E$ $\sim(1-E) \sim \underline{1}_{\Re}$ in $\Re[7,4.5]$.

Therefore $\mathfrak{N} \subset \mathfrak{A}$ by Theorem 2 . It remains to show that each type $I_{n}$ $(n<\infty)$ summand of $\mathcal{C}$ belongs to $\mathcal{Q}$. This can be done in a rather obvious way using the fact that such an algebra has the form $\mathscr{Z} \otimes M_{n}$, where $\mathscr{Z}$ is the centre of the algebra and $M_{n}$ is the algebra of $n \times n$-matrices. We do not enclose this proof in detail.

REMARK 1. It is a question of special interest to decide whether a local $C^{*}$ algebra is complete if one knows in addition that all of its maximal abelian star algebras are $W^{*}$ - or $A W^{*}$-algebras. We call such algebras local $W^{*}$ - and local $A W^{*}$-algebras respectively (in the first case we think of the algebra as represented on Hilbert space).

(a) If we could show that every local $W^{*}$-algebra $\mathcal{Q}$ is complete, then we could use Pedersen's theorem in [8] to show that $\mathbb{Q}$ is even a $W^{*}$-algebra. 
(b) $A W^{*}$-algebras are characterized among all $C^{*}$-algebras by axioms referring to the structure of their abelian star subalgebras only. If we could show that every local $A W^{*}$-algebra is a $C^{*}$-algebra, then we could characterize $A W^{*}$-algebras among all normed involutive algebras entirely by their abelian star subalgebras.

Following the lines of [7] it is easy to develop a structure theory for local $A W^{*}$-algebras in analogy to the structure theory for $A W^{*}$-algebras and, in particular, to define what a properly infinite local $A W^{*}$-algebra is. Then the proof of Theorem 2 immediately shows: Every properly infinite local $A W^{*}$. algebra is complete.

Unfortunately, we do not know anything about the type $\mathrm{II}_{1}$ case. We do not include the proof of the above result, since no new ideas are involved.

REMARK 2 . Let us call a $C^{*}$-algebra $C$ strongly interwoven, if every dense star subalgebra $\mathbb{Q}$ of $\mathcal{C}$ which contains with each $x=x^{*} \in \mathbb{Q}$ the $C^{*}$-algebra generated by $x$, is complete. $\mathcal{K}(\mathcal{H})$ is not strongly interwoven as the example of the dense subalgebra of all operators of finite rank on $\mathcal{H}$ shows. It does not seem unlikely, however, that $\mathscr{B}(\mathscr{F})$ or $\mathscr{B}(\mathcal{H C}) / \mathscr{K}(\mathcal{K} C$ ) are strongly interwoven.

\section{REFERENCES}

1. H. Behncke, A note on the Gel'fand-Naimark conjecture, Comm. Pure Appl. Math. 23 (1970), 189-200. MR 41 \#2404.

2. H. Behncke and H. Leptin, Classification of $C^{*}$-algebras with a finite dual, J. Functional Analysis 16 (1974), 241-257. MR 49 \#9638.

3. F. F. Bonsall and J. Duncan, Complete normed algebras, Springer-Verlag, Berlin and New York, 1973.

4. S. B. Cleveland, Homomorphisms of non-commutative *-algebras, Pacific J. Math. 13 (1963), 1097-1109. MR 28 \#1500.

5. J. Cuntz, Locally $C^{*}$-equivalent algebras, J. Functional Analysis 23 (1976), 95-106.

6. - On the continuity of semi-norms on operator algebras, Math. Ann. 220 (1976), 171-183.

7. I. Kaplansky, Projections in Banach algebras, Ann. of Math. (2) 53 (1951), 235-249. MR 13, 48.

8. G. K. Pedersen, Operator algebras with weakly closed abelian subalgebras, Bull. London Math. Soc. 4 (1972), 171-175. MR 47 \#9300.

9. J. R. Ringrose, Linear functionals on operator algebras and their Abelian subalgebras, J. London Math. Soc. (2) 7 (1974), 553-560. MR 49 \#1142.

FakUltät für Mathematix, Universität OSNabrüCK, FACHbereich 5, 4500 OsNabrüCK, Federal Republic of Germany (Current address of H. Behncke)

Fakultät für Mathematix, Universität Biblefeld, 4800 Bielefeld, Federal Republic of GERMANY

Current address. (J. Cuntz): Fachbereich Mathematik, Technische Universität Berlin, Strasse des 17. Juni 135, 1000 Berlin 12, Federal Republic of Germany 